\title{
Quality of Life and Meeting 24-h WHO Guidelines Among Preschool Children in Singapore
}

\author{
Michael Yong Hwa Chia ${ }^{1}$ Lee Yong Tay $^{2} \cdot$ Terence Buan Kiong Chua ${ }^{1}$
}

Published online: 3 October 2019

(c) The Author(s) 2019

\begin{abstract}
The objective of the study was to determine the proportion of preschool children under 5 years old meeting the 24-h World Health Organisation guidelines on physical activity, sedentary behaviour and sleep. Another objective was to examine the association between the health-related quality of life and meeting these guidelines among preschool children in Singapore. Parents completed an online and anonymous SMALLQ ${ }^{\circledR}$ (Surveillance of digital Media hAbits in earLy chiLdhood Questionnaire) on children's physical activity, sedentary behaviour and sleep. Meeting the 24-h WHO guidelines meant, within a 24-h period, (i) having at least $180 \mathrm{~min}$ of physical activity (ii) engaging in less than $60 \mathrm{~min}$ of screen media and (iii) having 10-13 h of good quality sleep. Parent-reported health-related quality of life of the children was determined using the Pediatric Quality of Life Inventory ${ }^{\mathrm{TM}}$, collected online at the same time. Parent-reported data showed that $12.6 \%$ met none while $9.6 \%$ of preschool children met all the WHO guidelines. $70.7 \%, 56.9 \%$ and $26.5 \%$, of preschool children respectively, achieved the sleep, physical activity and screen media use guidelines within a 24-h period. $40.5 \%$ met two guidelines while $37.4 \%$ met one guideline. Significant differences were detected in the health-related quality of life among preschool children who met all, none, or met $1-2$ of the WHO guidelines (i.e. total health score: $82.9 \pm 12.4$ vs. $76.4 \pm 15.1$ vs. $78.6 \pm 14.5 \%$, $\left.\mathrm{p}<0.05 ; \mathrm{\eta}^{2}=0.008-0.11\right)$. Our results show that the health-related quality of life of preschool children increased with the number of WHO guidelines accomplished.
\end{abstract}

Keywords SMALLQ ${ }^{\circledR} \cdot$ World Health Organisation guidelines $\cdot$ Quality of life $\cdot$ Preschool children

\section{Introduction}

Sleep, sedentary behaviour and physical activity are normal activities of children less than 5 years old over $24 \mathrm{~h}$. Systematic reviews show that less sedentary time, more physical activity and sufficient sleep are essential ingredients for the healthy growth and development of children aged below 5 years (Carson et al. 2017; Chaput et al. 2017a, b) More research is focused on physical activity in relation to health outcomes, usually obesity (Carson et al. 2017) or sleep

Michael Yong Hwa Chia

michael.chia@nie.edu.sg

1 Physical Education and Sports Science Academic Department, National Institute of Education, Nanyang Technological University, 1 Nanyang Walk, Singapore 637616, Singapore

2 Office of Education Research, National Institute of Education, Nanyang Technological University, 1 Nanyang Walk, Singapore 637616, Singapore
(Chaput et al. 2017a, b) or in terms of sedentary behaviour and health outcomes (Poitras et al. 2017). The associations between all three behaviours-physical activity, sedentary behaviour and sleep, together or in combination, and health outcomes is less studied (Carson et al. 2017). Yet it is important to consider all three behaviours over a 24-h period, in relation to health, since each of these behaviours, repeated daily, are habit-forming, and are likely to become lifestyle habits in later childhood and adolescence (Stiglic and Viner 2019).

\section{Integrated 24-h Guidelines on Physical Activity, sedeNtary Behaviour and Sleep}

In Singapore, there are no guidelines for physical activity, sedentary behaviour and sleep, integrated as one over a 24-h period for preschool children. Age-specific guidelines for sleep for preschool children aged 3-5 years are available and mirror those outlined by the American Academy of Pediatrics, where $10-13 \mathrm{~h}$, in a $24-\mathrm{h}$ period including naps are 
recommended as the norm and that children should have regular bedtimes and a regular sleep routine (Respiratory Medicine Service, KK Women's and Children's Hospital). Guidelines on physical activity for Singapore by the Health Promotion Board of Singapore (2013) remain unchanged in 2019. The World Health Organisation WHO (2019) published and promoted integrated guidelines on physical activity, sedentary behaviour and sleep. Specifically, for children aged between 2 and 5 years, these guidelines stipulate:

- Children should be engaged in at least 180 min of physical activity, of which at least $60 \mathrm{~min}$ is moderate-to vigorous intensity physical activity, spread throughout the day, where more is better.

- Children should not be restrained for more than $60 \mathrm{~min}$ at a time or sit for extended periods.

- Sedentary screen time should be no more than $60 \mathrm{~min}$, where less is better. When sedentary, reading and storytelling with a caregiver is encouraged.

- Children should have 10-13 h of good quality sleep, which could include a nap and have regular sleep and wake times.

The Pediatric Quality of Life 4.0 Inventory (PedsQL ${ }^{\mathrm{TM}}$ $4.0)$ is widely used to assess general health-related quality of life of children and adolescents aged 2-18 years in healthy and diseased conditions (Varni et al. 1999, 2003). In the United Kingdom, the parent-reported PedsQL ${ }^{\mathrm{TM}}$ in 256 healthy toddlers (aged 2-4 years) is reported as suitable and acceptable for use with good internal consistency $(>0.70)$ (Buck 2012). Studies using the PedsQL ${ }^{\mathrm{TM}}$ in Asian populations of young children aged 2-4 years are limited. However, the quality of life using the parent-reported PedQL ${ }^{\mathrm{TM}}$ was profiled in 939 preschool children (aged 2-4 years) with eyesight problems in Singapore (Lamoureux et al. 2010). Research on 336 overweight and obese children aged 2-7 years in Hong Kong using the PedsQL ${ }^{\mathrm{TM}}$ was reported (Chan and Wang 2013). The two cited studies produced mixed results - the total health scores were unaffected in Singaporean preschool children with refractive errors while overweight, obese and underweight children living in Hong Kong had lower scores on health-related quality of life than normal weight peers.

The association between the combined effects of meeting or not meeting the 2019 WHO guidelines on physical activity, sedentary behaviour and sleep, on the health-related quality of life using the PedsQL ${ }^{\text {TM }}$ is unknown. We hypothesized that the parent-reported health quality of life of preschool children has an association with meeting the guidelines for physical activity, sedentary behaviour and sleep. This association, we hypothesized is mediated through the socio-demographic characteristics of parents. The specific research questions were:
1. To what extent did preschool children meet guidelines for (a) physical activity, (b) sedentary behaviour, and (c) sleep?

2. What was the association between the health-related quality of life and meeting the WHO guidelines on physical activity, sedentary behaviour and sleep for children under 5 years?

3. Did parent sociodemographic characteristics explain the extent to which children met the WHO guidelines on physical activity, sedentary behaviour and sleep?

\section{Methods}

\section{Ethical Clearance, Recruitment and Informed Consent}

Institutional ethics clearance for the research was granted (IRB-2017-09-036). Parents of preschool children, aged 2-4 years were contacted via the anchor operators of preschool centres in Singapore. Four out of five anchor operators in Singapore collaborated with the university researchers in the iPreschooler study-a tracking study of screen media use in preschool children over 36 months in Singapore. Singapore is a state and city-all locations in Singapore are urban. The Anchor Operator scheme-funded by the government-provides funding support to selected preschool operators to increase access to good quality and affordable early childhood care and education, especially for children from lower income or disadvantaged backgrounds (Early Childhood Development Agency 2019). Parent participants who enrolled children in these preschools provided informed consent to voluntary participation and were assured of anonymity. The researchers abided by all conditions stipulated in the Singapore Personal Data Protection Act (2012) throughout the study. Overall, the number of preschool centres and kindergartens involved in the research numbered 425 .

\section{Instrument}

An online survey called Surveillance of digital Media hAbits in earLy chiLdhood Questionnaire (SMALLQ ${ }^{\circledR}$ ) (Chia et al. 2019) and the Pediatric Quality of Life Inventory (Varni et al. 1999) were hosted on a secure platform called Qualtrics that was approved by the university.

\section{Surveillance of screen Media hAbits in earLy chiLdhood Questionnaire (SMALLQ ${ }^{\circledR}$ )}

SMALLQ ${ }^{\circledR}$ is a registered trademark (40201824154R) until 2028. The development of SMALLQ ${ }^{\circledR}$ and its validity are reported elsewhere (Chia et al. 2019). SMALLQ ${ }^{\circledR}$, consists 
of 25 questions and is based in part, on the conceptual understanding of digital media parenting outlined by O'Connor et al. (2013). Essentially, this means framing questions on screen media use in the domains of content (e.g. for education or for entertainment; age-appropriate content or not), context (e.g. whether parent is present; how is screen media delivered-computer, smart phone, game console) and dose (e.g. how much is each screen media used). Further, the AMEE framework-Guide No. 87 (Artino et al. 2014) guided the development of the SMALLQ ${ }^{\circledR}$. A unique feature of the process was that it blended the views of parents and that of experts, thereby front-loading the validity of responses via careful item selection. Specifically, the SMALLQ ${ }^{\circledR}$ instructed a parent to provide data on his/her child on a typical weekend day, outside of preschool via a seven-day recall.

\section{Screen Media Environment at Home}

Information about the type of technological devices that children accessed at home was solicited. These included fixed screens-television, desktop computers and game consoles, and mobile screens-smartphones, tablets and other hand-held devices and technological toys.

\section{Parent Screen Media Use}

Information about parental screen media habits on a typical weekend day was solicited. The choices were segregated as use for work, entertainment, social networking and for personal development. Screen media use meant time spent accessing content transmitted via the internet or any computer or TV network.

\section{Screen Media Use of the Child Outside of Pre-school/ Kindergarten}

Parents were asked to provide details of children's screen media habits outside of the pre-school/kindergarten, on a typical weekend day. This included the frequency, duration and purpose of screen media use (e.g. for learning, for entertainment, for communication) on fixed screens, and on mobile screens.

\section{Parent Perception of Screen Media}

Parents were asked to indicate their perceived importance of screen media in relation to them and to children using a 5-point Likert scale from 'not important' to 'very important'. Options for the benefit to the child include 'Improve knowledge and skills', 'Entertainment', and 'Communication'. Options for the benefit to parent include 'Keep child occupied', 'Distract or divert the attention of the child' and 'Put the child to sleep'.

\section{Parental Concern of Children Screen Media Use}

Parents reported perceived concerns on screen media use of children on a 5-point Likert scale from 'not concerned' to 'seriously concerned'. Options for parents included 'Poor sleep', 'Poor eyesight', 'Lack of physical exercise or play', 'Exposure to inappropriate content', 'Addiction', and 'Lack of parent-child interaction'.

\section{Parental Awareness and Enforcement of Children Screen Media Use Guidelines}

Parents indicated whether they were aware of guidelines on screen media use by children and if they enforced those guidelines. The guidelines are (i) limit screen time to $60 \mathrm{~min}$ per day (ii) introduce high-quality educational programmes (iii) co-watch or co-play with child when engaged in screen media.

\section{Non-screen Habits of the Child Outside of Pre-school/Kindergarten}

Parents reported on children's physical activity and play on a typical weekend day outside of the preschool. Information on the duration spent on various indoor activities (nonscreen reading and drawing), duration of indoor play and outdoor play, and an estimated apportion of time the child spent on moderate-to-vigorous activities, on a typical weekend day, was obtained.

\section{Demographic and Other Information}

The SMALLQ ${ }^{\circledR}$ also included demographic questions about the parent (e.g. relationship to the child, monthly household income, highest educational attainment, and ethnicity) and other information on the child (e.g., gender, age, height and weight, sleep duration, and sleep quality).

\section{The Pediatric Quality of Life 4.0 Inventory (PedsQL ${ }^{\mathrm{Tm}}$ 4.0)}

The acute 7-day recall version of the PedsQL ${ }^{\mathrm{TM}} 4.0$ Inventory (1998) was used to assess the parent-reported health-related quality of life of children. The 21-item questionnaire yielded a total health score and scores on four separate scales. These scales sought parents' input on the level of difficulty that children faced with (a) Physical Functioning (eight items), Emotional Functioning (five items), Social Functioning (five items) and School Functioning (three items). Each subscale was based on a 5-point Likert scale (i.e. never, almost never, 
sometimes, often, and almost always a problem). The questionnaire items were scored as 0, 25, 50, 75 and 100. Scale scores were calculated by dividing the sum of item scores by the number of items. The Physical Health Summary Score is the same as the physical functioning scale. Examples for physical functioning are walking more than a block, running, lifting something heavy or having a low energy level. The Psychosocial Health Summary Score corresponds to sum of the means of the Emotional, Social and School Functioning scales. Examples for emotional functioning are feeling afraid or feeling angry; examples for social functioning are getting along with other children or keeping up when playing with other children; examples for school functioning are paying attention in class, or missing school because of illness. The total health score (0-100) is the sum of Psychosocial Health (composite of Social, Emotional and School Functioning scales) and Physical Health (Physical Functioning scale). Scores ranged from 0 to 100 , with a higher score indicating a better quality of life. The PedsQL ${ }^{\text {TM }} 4.0$ is feasible and reliable, is a well-validated instrument in healthy populations and demonstrates cross-cultural applicability (Varni et al. 1999, 2003). In the present study, the internal consistency for the parent-reported PedsQL ${ }^{\text {TM }} 4.0$ was considered high to excellent- Cronbach alpha for Total Health was 0.91; for Psychosocial Health was 0.86 and for Physical Health was 0.88 . These findings suggested that the PedsQL ${ }^{\text {TM }} 4.0$ generated for the Singaporean dataset are reliable.

\section{Limitations of the Use of Questionnaire and Mitigations}

Self-reported questionnaires are prone to social desirability bias, and with problems of recall. Social desirability bias simply explained, is the tendency for people to answer questions in surveys that appear to be socially acceptable by the popular view. For example, in the SMALLQ ${ }^{\circledR}$, a segment polled parent awareness and practice of screen media guidelines. Parents may over-report that they know what the guidelines are and that they practised those guidelines (e.g. limit child screen media use to no more than 60 min daily) (Chung and Monroe 2003). In terms of recall, parents may not be able to recall accurately the amount of time spent engaged in screen media for themselves and for their child. While social desirability bias and accurate recall are plausibly limitations in the use of the online SMALLQ ${ }^{\circledR}$, there were several mitigating factors that could 'limit these limitations'. To minimize social desirability bias, the online survey is anonymous and there are instructions for completing the survey stating that there are no right or wrong answers at the beginning of the survey. Further, the questionnaire items included statements that asked parents to choose the options that best described behaviours and beliefs that were common viewpoints (e.g. importance of screen media to child and/or to themselves). To mitigate recall problems, notes that were provided within the SMALLQ ${ }^{\circledR}$ guided parents on how to answer questionnaire items, and helped with a more accurate recall of questions posed-for example, in the last 7 days, when your child used media, "estimate the amount of time you were engaged with him/her-while watching videos together' or 'take the total time your child spent using screen media to be $100 \%$. This applied to recall questions on time spent on screen media, non-screen media, sleep and play. All recall-in the SMALLQ ${ }^{\circledR}$ and PedsQL ${ }^{\text {TM }} 4.0$-was limited to the last 7 days. The literature affirms the usefulness and reliability of using shorter timeframe for recall in healthrelated surveys, in comparison to a monthly or yearly basis. Even then, this would depend on the context of the research (Short et al. 2009). Moreover, the use of internet-based surveys (i.e. SMALLQ ${ }^{\circledR}$ and PedsQL ${ }^{\mathrm{TM}}$ )—were hosted on a secured online platform, Qualtrics-are reported as a useful and an effective method of collecting self-reported or proxy data in health-related research (Palmer et al. 2012).

\section{Data Collection and Statistical Analysis}

Data were collected in Singapore between March and August of 2018. For each anchor operator, the online questionnaire was active for a period of 10 days. This meant that when parents consented to participate and complete the online survey, the data on Qualtrics were 'captured' at the backend. On the $5^{\text {th }}$ day of data-collection, an online notification was sent to all parents through the anchor operators, to remind parents to complete the questionnaires. This approach was adapted from the Total Design Method outlined by Hoddinott and Bass (1986), which helped to increase participation. Responses to each of the online survey questions were not mandatory to avoid any form of coercion. The number of completed responses to each survey question was recorded.

Parent-reported child screen media use in each activity (i.e. using screen media for education, using screen media for entertainment, creating media and communicating) were summed to derive the total child screen media use. Total child non-screen-based sedentary time was derived from the combined time spent on sedentary activities without the use of a screen (i.e. reading printed books, drawing and other forms of craftwork and helping with simple household chores).

\section{Parametric Tests}

Descriptive statistics for the proportion of children who met and did not meet the physical activity, screen time and sleep were generated-meeting all three guidelines, meeting 1-2 of the guidelines, and meeting none of the 24-h WHO guidelines. Differences in (a) the duration of physical play, sedentary activities and sleep, and (b) quality of life 
scores between children who met all three guidelines and those who met none of the guidelines, were examined using independent-sample t-tests. The measure of effect size was reported as partial eta square, where $\eta^{2}=0.01$ represented small effect size, $\eta^{2}=0.06$ represented a medium effect size and $\eta^{2}=0.14$ represented a large effect size.

\section{Non-parametric Tests}

The associations between parent characteristics (i.e. household income and education level) and the number of guidelines on physical activity, sedentary behaviour and sleep met by preschool children were assessed using Chi square tests for associations. The strength of these associations is reported as Phi values, where 0 means no relationship; $0-.2$ is weak, .2-.3 is moderate and . 3 and above is strong. Sleep quality was analysed using the Kruskal-Wallis U-test. Statistical significance was set at $p<0.05$.

\section{Results}

A main objective of the study was to describe parentreported data that show the proportion of children who met the 2019 WHO guidelines on physical activity, sedentary behaviour and sleep, Table 1 shows (a) the percentage of preschool children who met each of the daily guidelines on physical activity (at least $180 \mathrm{~min}$ ), sedentary behaviour (less than $60 \mathrm{~min}$ of screen media use) and sleep (10-13 h); and (b) the percentage of those who met a number of the guidelines (i.e. all, none, one or two).

More of the preschool children met the sleep guideline (70.7\% had $10-13 \mathrm{~h}$ of total sleep), followed by physical activity (56.9\% had $180 \mathrm{~min}$ ), while $26.5 \%$ of the preschool children were in compliance with the guideline of limiting daily screen media use to no more than $60 \mathrm{~min}$.
More preschool children met none than met all the 24-h WHO guidelines.

Figure 1 presents a Venn diagram showing the interactions of the 24-h WHO guidelines on physical activity, sedentary behaviour and sleep met by the preschool children.

Table 2 provides descriptive information on physical activity, screen media use and sleep on a typical weekend day.

Table 3 provides the parent characteristics in relation to preschool children who met all, 1-2 and met none of the WHO guidelines on physical activity, sedentary behaviour and sleep.

The PedsQL ${ }^{\mathrm{TM}}$ scores for preschool children who met all $3 \mathrm{WHO}$ guidelines were significantly greater than those who met 1-2 guidelines and those who met none of the guidelines $(\mathrm{p}<0.05)$. In terms of total health, psychosocial health and physical health, respectively, these were $8.5 \%, 7.4 \%$ and $9.6 \%$ higher, respectively for preschool children who met all $3 \mathrm{WHO}$ guidelines compared to those who met none of the guidelines, with partial eta effect sizes hovering at the medium level for the comparisons of differences between the two extreme ends of the spectrum.

Table 4 provides a summary of children's PedsQL ${ }^{\text {TM }}$ scores in relation to meeting the number of WHO guidelines on physical activity, sedentary behaviour and sleep.

The ethnicity of parents surveyed in the present study are similar to that reported in the national population census reported by the Department of Statistics Singapore (2010). About $75 \%$ of the parent respondents were mothers, followed by fathers and then, legal guardians, which was a small percentage. Survey data on male and female preschool children were about equal. The associations between: (a) number of guidelines met and monthly household income, and (b) number of guidelines met and parental education level were significant, where Chi square $\chi^{2}(15)=29.852, p=0.012$,

Table 1 Percentage of preschool children meeting WHO guidelines

\begin{tabular}{|c|c|c|c|c|}
\hline \multirow[t]{2}{*}{ 24-h World Health Organisation guidelines } & \multicolumn{2}{|l|}{ Met } & \multicolumn{2}{|c|}{ Did not meet } \\
\hline & Valid \% & $\mathrm{N}$ & Valid \% & $\mathrm{N}$ \\
\hline 180 min of physical activity & 56.9 & 1282 & 43.1 & 973 \\
\hline 10-13 h of sleep (including naps) & 70.7 & 1602 & 29.3 & 663 \\
\hline Screen media time of $60 \mathrm{~min}$ or less & 26.5 & 621 & 73.5 & 1725 \\
\hline \multirow[t]{2}{*}{ All of the above guidelines } & 9.6 & 228 & 90.4 & 2156 \\
\hline & \multicolumn{2}{|c|}{ Valid \% } & & $\mathrm{N}$ \\
\hline \multicolumn{5}{|c|}{ Parent-reported number of guidelines met by preschool children } \\
\hline None & \multicolumn{2}{|c|}{12.6} & & 300 \\
\hline 1 & \multicolumn{2}{|c|}{37.3} & & 891 \\
\hline 2 & \multicolumn{2}{|c|}{40.5} & & 965 \\
\hline 3 & \multicolumn{2}{|c|}{9.6} & & 228 \\
\hline
\end{tabular}


Fig. 1 Venn diagram illustrating proportion of children meeting 24-h WHO guidelines
Table 2 Mean \pm SD for physical play, screen media and sleep of child on a typical weekend day

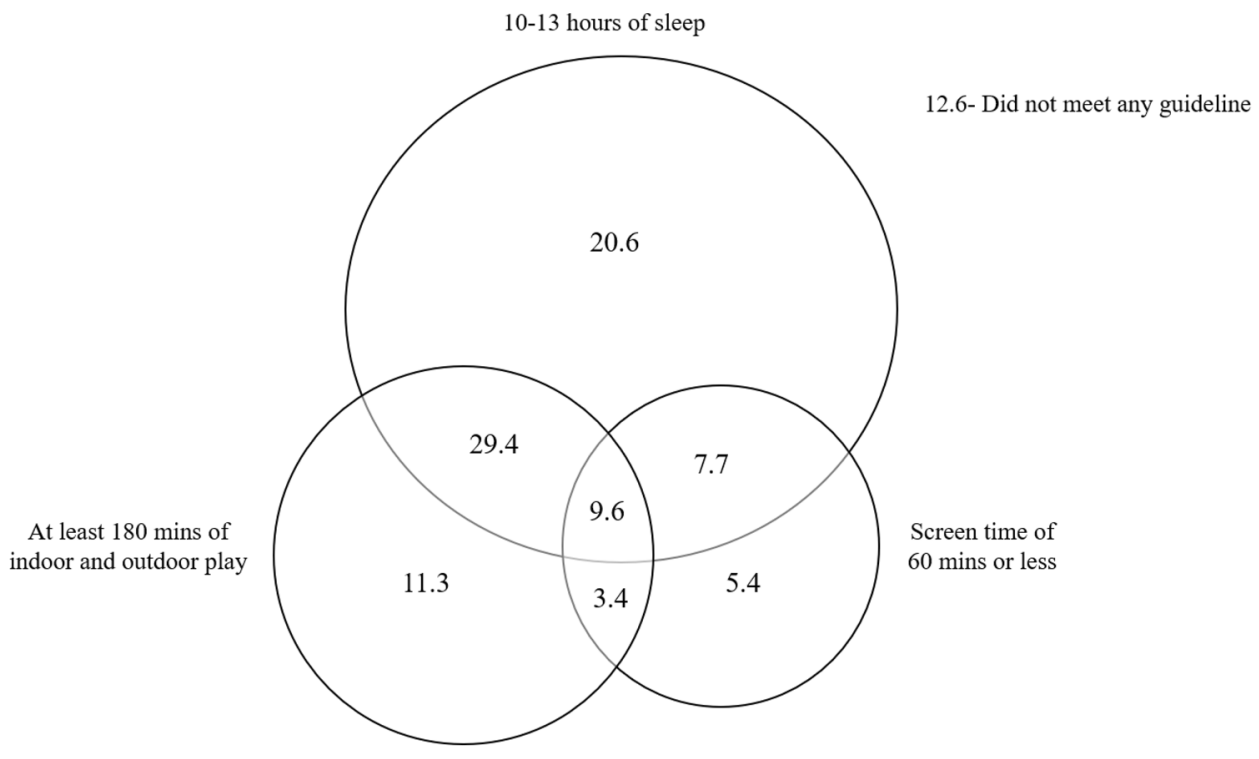

\begin{tabular}{|c|c|c|c|c|}
\hline & $\begin{array}{l}\text { Meet all } 3 \\
\text { guidelines } \\
(9.6 \%)\end{array}$ & $\begin{array}{l}\text { Meet } 1-2 \text { guide- } \\
\text { lines }(77.8 \%)\end{array}$ & $\begin{array}{l}\text { Did not meet any } \\
\text { guideline }(12.6 \%)\end{array}$ & $\eta^{2}$ \\
\hline \multicolumn{5}{|l|}{ Physical activity (h) } \\
\hline Indoor and outdoor play & $4.7 \pm 1.5^{* \dagger}$ & $3.4 \pm 1.9^{*}$ & $1.6 \pm 0.7$ & 0.131 \\
\hline Energetic play & $2.6 \pm 1.5^{* \dagger}$ & $1.8 \pm 1.4 *$ & $0.7 \pm 0.5$ & 0.081 \\
\hline \multicolumn{5}{|l|}{ Sedentary behaviour (h) } \\
\hline Screen media activities ${ }^{\mathrm{a}}$ & $0.7 \pm 0.3 *^{\dagger}$ & $2.6 \pm 1.7 *$ & $3.0 \pm 1.4$ & 0.122 \\
\hline Non-screen media activities ${ }^{b}$ & $1.6 \pm 0.9$ & $1.8 \pm 1.2 *$ & $1.4 \pm 1.1$ & 0.008 \\
\hline \multicolumn{5}{|l|}{ Sleep $(h)$} \\
\hline Duration $^{c}$ & $11.5 \pm 0.9 * \dagger$ & $10.6 \pm 3.0^{*}$ & $7.0 \pm 5.3$ & 0.113 \\
\hline Quality (valid \%) ${ }^{\mathrm{d}}$ & & & & $\begin{array}{l}0.011 \\
\quad(p=0.425)^{\beta}\end{array}$ \\
\hline Unsatisfactory & 0.4 & 0.7 & 1.4 & \\
\hline Below average & 4.0 & 3.2 & 4.7 & \\
\hline Average & 26.3 & 27.5 & 30.6 & \\
\hline Above average & 37.7 & 36.5 & 34.0 & \\
\hline Excellent & 31.6 & 32.1 & 29.3 & \\
\hline
\end{tabular}

$\eta^{2}$ denotes partial eta squared, where 0.01 is small effect size, 0.06 is medium effect size and 0.14 is large effect size

${ }^{\text {a}}$ Energetic play is explained as physical play that causes the child to 'huff and puff' (i.e. of moderate-tovigorous intensity)

${ }^{b}$ Non-screen based sedentary activities such as reading printed books, drawing and other forms of craftwork, and helping with household chores (excludes physical indoor play like board games)

${ }^{c}$ Duration of night-time sleep and daytime naps

${ }^{\mathrm{d}}$ Quality of sleep where unsatisfactory is when sleep is disrupted, there are nightmares and child has difficulty waking up in the morning; excellent is where the child falls easily to sleep at night, the sleep is uninterrupted and child wakes up refreshed in the morning

*Denotes a significant difference $(p<0.05)$ in duration compared to children who did not meet any guideline

${ }^{\dagger}$ Denotes significant difference $(p<0.05)$ in duration between children who met all 3 guidelines and those who met 1-2 guidelines

${ }^{\beta}$ Rosenthal's $r$ where 0.1 is small effect size, 0.3 is medium effect size and 0.5 is as large effect size 
Table 3 Parent and child characteristics and WHO guidelines
Table 4 Quality of life of children and meeting 24-h WHO guidelines

\begin{tabular}{|c|c|c|c|c|c|c|}
\hline & \multicolumn{2}{|c|}{ Met all 3 WHO guidelines } & \multicolumn{2}{|c|}{ Met 1-2 WHO guidelines } & \multicolumn{2}{|c|}{$\begin{array}{l}\text { Did not meet any } \\
\text { WHO guideline }\end{array}$} \\
\hline & Valid \% & $N$ & Valid \% & $N$ & Valid \% & $N$ \\
\hline \multicolumn{7}{|l|}{ Child characteristics } \\
\hline \multicolumn{7}{|l|}{ Gender } \\
\hline Male & 53.1 & 121 & 48.8 & 822 & 45.1 & 69 \\
\hline Female & 46.9 & 107 & 51.2 & 863 & 54.9 & 84 \\
\hline \multicolumn{7}{|l|}{ Parent characteristics } \\
\hline \multicolumn{7}{|c|}{ Relationship with child } \\
\hline Mother & 78.9 & 179 & 71.8 & 1209 & 75.2 & 112 \\
\hline Father & 21.1 & 48 & 27.4 & 462 & 22.8 & 34 \\
\hline Guardian & 0 & 0 & 0.8 & 13 & 2.0 & 3 \\
\hline \multicolumn{7}{|c|}{ Monthly household income } \\
\hline$\$ 0-\$ 8000$ & 57.1 & 129 & 65.9 & 1104 & 72.2 & 104 \\
\hline$\$ 8001$ and above & 42.9 & 97 & 34.1 & 571 & 27.8 & 40 \\
\hline \multicolumn{7}{|c|}{ Highest educational attainment } \\
\hline Below university & 30.8 & 70 & 45.7 & 766 & 50.3 & 75 \\
\hline University & 69.2 & 157 & 54.3 & 913 & 49.7 & 74 \\
\hline \multicolumn{7}{|l|}{ Ethnicity } \\
\hline Chinese & 76.2 & 173 & 67.5 & 1134 & 64.2 & 95 \\
\hline Malay & 11.0 & 25 & 14.5 & 244 & 16.2 & 24 \\
\hline Indian & 8.8 & 20 & 11.5 & 193 & 12.8 & 19 \\
\hline Eurasian & 0.5 & 1 & 0.3 & 5 & 0 & 0 \\
\hline Others & 3.5 & 8 & 6.2 & 104 & 6.8 & 10 \\
\hline
\end{tabular}

\begin{tabular}{lllll}
\hline $\begin{array}{l}\text { Child quality of life score } \\
\text { (out of 100) }\end{array}$ & $\begin{array}{l}\text { Met all 3 guidelines } \\
(9.6 \%)\end{array}$ & $\begin{array}{l}\text { Met 1-2 guidelines } \\
(77.8 \%)\end{array}$ & $\begin{array}{l}\text { Did not meet any guide- } \\
\text { line }(12.6 \%)\end{array}$ & $\eta^{2}$ \\
\hline Total health & $82.9 \pm 12.4^{* \dagger}$ & $78.6 \pm 14.5$ & $76.4 \pm 15.1$ & 0.11 \\
Psychosocial health & $79.5 \pm 13.6^{* \dagger}$ & $75.9 \pm 14.4$ & $74.0 \pm 14.6$ & 0.008 \\
Physical health & $87.5 \pm 15.8^{* \dagger}$ & $83.0 \pm 18.7^{*}$ & $79.8 \pm 19.8$ & 0.008 \\
\hline
\end{tabular}

$\eta^{2}$ denotes partial eta squared, where 0.01 is small effect size, 0.06 is medium effect size and 0.14 is large effect size

*Denotes a significant difference $(p<0.05)$ compared to children who did not meet any guideline

${ }^{\dagger}$ Denotes a significant difference $(p<0.01)$ between child who met all 3 guidelines and those who met $1-2$ guidelines

$\varphi=0.121$ and $\chi^{2}(15)=57.671, p=0.001, \varphi=0.167$, respectively.

\section{Discussion}

\section{Characteristics of Present Research}

The present study involved a large dataset of parent-reported information on children who attend preschool and kindergarten in Singapore. In terms of ethnicity, it was close in representation to the Singapore population. The proportion of university-educated parents ranged from 50 to $69 \%$ in the present research, which, according to Short et al. (2009) tend to provide more accurate data in online surveys on healthcare associated issues. The proportion of university-educated parents was higher than the national average for Singapore since the parents represented in the poll belonged to the younger generation. In all likelihood, the younger generation of parents are more exposed to and comfortable with screen media (Prensky 2001).

\section{Research Objectives}

The objectives of the study, were (a) to explain the extent preschool children in Singapore, are in compliance to the integrated 24-h WHO guidelines on physical activity, sedentary behaviour and sleep, (b) how meeting the 24-h WHO 
guidelines was related to children's health-related quality of life, and (c) to what extent were parent educational level and household income associated with meeting the 24-h WHO guidelines on physical activity, sedentary behaviour and sleep.

\section{Meeting the WHO Guidelines}

From Table 1, more children met the guideline of having $10-13 \mathrm{~h}$ of sleep over a $24-\mathrm{h}$ period than for physical activity, including at least $60 \mathrm{~min}$ of energetic play but only a small percentage was in compliance with limiting screen media use to not more than 60 min a day. At both ends of the spectrum-meeting all three and meeting none of the guidelines-a higher percentage met none of the guidelines than those who met all of the guidelines. This is concerning for Singapore as the prevalence of this group of children may increase with age, and, over time, with inimical consequences all round (Carson et al. 2017; Chaput et al. 2017a, b; Poitras et al. 2017). From Table 2, preschool children who met all the WHO guidelines had a significantly longer playtime (including energetic play), less screen media use, and slept longer than those who met none of the guidelines.

\section{Research Comparisons}

These abovementioned findings compare well, but are not identical to the Canadian results reported by Carlson et al. (2017). For instance, a higher percentage of preschool children in Singapore met all the guidelines (i.e. 9.6 vs. 5\%) but more preschool children in Singapore met none of the guidelines (i.e.12.6 vs. 6.1\%) compared to the findings of the cited study. The present results contrasted to results of other studies where higher proportions of preschool children (12.7-14.9\%) in Canada (Chaput et al. 2017a, b) and Australia (Cliff et al. 2017) respectively, met all the 24-h WHO guidelines.

\section{Physical Activity Guideline}

Preschool children in Singapore were better able to accomplish this guideline compared to preschool children in Canada and Australia. In the case of the Canadian study (Carlson et al. 2017), the physical activity requirement, was least accomplished by the preschool children. These differences are perhaps attributed to the differences in the methods used for estimating the physical activity between the two studies. In the Canadian research, physical activity was assessed using accelerometers whereas physical activity in the present research was parent-reported using an online survey. Nonetheless, objective and subjective assessments of physical activity are not immune to errors so when these data are compared, interpretations should be made with caution (Chia 2008). Weather and sociocultural differences between the two countries may also contribute to the different findings. For instance, Harrison et al. (2017) showed that accelerometer-determined physical activity of children varied with the daylight hours and weather conditions. They summarised that children from Northern European countries and Melbourne, Australia, were the most active, and better maintained their activity levels given the weather conditions they experienced compared to those in the United States and Western Europe. Singapore is geographically located close to the Tropics with uniform hours of daylight and a stable temperature range throughout the year. This may explain a relatively higher proportion of preschool children meeting the daily guideline of 180 min of physical activity in the present research.

\section{Sleep Guideline}

Similar studies show that among preschool children, the proportions of those meeting the $10-13 \mathrm{~h}$ of night-time sleep are greater than those reported in the present research (see Table 1). For instance, Tamana et al. (2019) reported that $84 \%$ of the 2427 children polled at 5 years old had $10-13 \mathrm{~h}$ of parent-reported night-time sleep, with other studies showing a range of 83.9-88.7\% (Chaput et al. 2017a, b; Cliff et al. 2017) of children meeting the sleep guideline. Differences in sleep among preschool children are an interplay between 'culture (and therefore behaviour) and biology' in explaining children's sleep (Jenni and O'Connor 2005). They cited Italian children aged 2-4 years having less night-time sleep than American children of the same age do. Italian children had no fixed bedtimes and often participated in evening social activities with adults and regularly fell asleep before they were put to bed according to New and Richman (1996). Liu et al. (2003) reported that Chinese schoolchildren had substantially shorter sleep durations than children in other cultures because of late bedtimes and early rise times for schooling. This shortened night-time sleep is reported in Japanese children too (Steger 2003). For both the Chinese and Japanese children, the reduced night-time sleep durations are attributed to the familial emphasis on studying and learning, and the pressures of academic achievement (Liu et al. 2003; Steger 2003). In the case of the present results, the night-time sleep of preschool children who met all three WHO guidelines was significantly longer than those who met none of the guidelines.

\section{Sedentary Behaviour Guideline}

In the present research, $26.5 \%$ of the preschool children met the guideline of limiting screen media use to $60 \mathrm{~min}$ or less over a 24 -h period. This compares with $51.7 \%$ reported by Carson et al. (2017) and 17.3-24.4\% reported by others 
(Chaput et al. 2017a, b; Cliff et al. 2017). These differences in results across the Singaporean, Canadian and Australian research findings may be explained by contextual and socioeconomic differences between the countries. This emphasizes the need to conduct similar research within different countries for useful country and culture-specific data. In the present study, screen media use for those who met none of the guidelines was 4.3 times ( 3.0 vs. $0.7 \mathrm{~h}$, see Table 2 ) that of those who met all the guidelines. Concomitantly, moderate-to-vigorous play in those who met none of the guidelines was $27 \%$ that of children who met all the guidelines. The demerits of excessive screen media use among preschool children in Singapore needs amelioration. For instance, a systematic review of reviews showed that for children and adolescents, the evidence for the negative impact of excessive screen time use was strongest for adiposity, unhealthy diet, depressive symptoms and quality of life (Stiglic and Viner 2019). Intervening early helps to reduce unhealthy screen habits from being entrenched in childhood and in adolescence and these interventions need to be relevant and sustainable.

\section{Health-Related Quality of Life and Meeting the WHO Guidelines}

From Table 3, a significant difference in quality of life scores for total health, psychosocial health and physical health for preschool children who met all three guidelines compared to those who met none of the guidelines, is apparent. Few studies have examined the relationship between health-related quality of life and screen media use among preschool children. Among the few, Puder et al. (2013) reported that when compared to non-migrant preschool children, preschool children from migrant families living in two different regions in Switzerland, have significantly lower PedsQL ${ }^{\mathrm{TM}}$ total score, in one region but not in the other. Importantly, Puder et al. (2013) reported that the most relevant confounders of their findings were parental education, paternal occupation, child screen time and child BMI. The screen time reported for children aged $5-7$ years was $31 \pm 30,37 \pm 35$ and $52 \pm 49 \mathrm{~min}$ per day, respectively for families with both parents who were non-migrants, one parent and both parents who were migrants. The associated PedsQL ${ }^{\mathrm{TM}}$ Total Health scores were $84.2 \pm 9.1,82.7 \pm 9.6$ and $81.7 \pm 11.7 \%$, respectively. In comparison, the Total Health Scores in the present study are $82.9 \pm 12.4,78.6 \pm 14.5$ and $76.4 \pm 15.1 \%$, respectively for preschool children who met all three, met 1-2 and met none of the guidelines stipulated by WHO on physical activity, sedentary behaviour and sleep. Also, the effect sizes were considered as moderate, where physical and psychosocial health, as was the Total Health score, was significantly different between the preschool children meeting all three guidelines versus meeting none of the guidelines. These results are in agreement to findings of a review of reviews conducted by Stiglic and Viner (2019), where a poorer quality of life in children and adolescents is featured as strong evidence of a negative impact for excessive screen media use.

\section{Parent and Home Characteristics and WHO Guidelines}

The present results show that preschool children who met none of the WHO guidelines tended to come from households with lower income brackets compared to those who met all three guidelines and with lower parental levels of education (see Table 3), albeit the effect sizes were weak. De Craemer et al. (2018) reported that a higher socioeconomic status was associated with less time spent on screen-based activities in children with maternal education seemingly, the most influential parent characteristic in relation to children's sedentary activities. Another study showed that in low-to-middle income countries, sedentary behaviour, is more strongly associated with income, than with parental education (Mielke et al. 2017). In the case of the present finding, it may be that more affluent parents were better able to provide less sedentary pursuits for their preschool children such as paying for physical activity sessions (e.g. swimming or dance classes, etc.). Parents with higher levels of education could be more discerning and aware of screen media use guidelines than those with lower education levels and were better able to regulate their children's use of screen media. However, these explanations may need to be confirmed in future qualitative research involving parents.

\section{Strengths and Limitations}

A key strength of the study is that the research involved a sizeable sample of parents of preschool children from the low-to-middle income segment of the population in Singapore. They were, in terms of the parent ethnicity and gender of the preschool children, typical of the national population of Singapore. It is apparently the first study to compare the 2019 WHO guidelines on Physical Activity, Sedentary Behaviour and Sleep, and its prevalence of adherence among preschool children in Singapore. A limitation of the study was that the data were gathered via online questionnaires, which involved subjective responses and the recall of parents. However, mitigation measures to reduce social desirability (online anonymous survey, appropriate development of the questionnaires), and recall bias (survey limited to a 7 -day recall) were in place. 


\section{Conclusions}

The research showed that $9.6 \%$ of preschool children met all three integrated 24-h guidelines on physical activity, sedentary behaviour and sleep and $12.6 \%$ met none of the guidelines. The health-related quality of life of preschool children were significantly higher for those who met more of the guidelines than those who met none with physical health affected the most. Efforts to educate parents of children in Singapore about these integrated guidelines to promote healthy growth and development of children in early childhood must be an actionable priority.

Acknowledgements Preschool Anchor Operators, E-Bridge, Skool4kidz, PCF Sparkletots Preschool and MY World, for partnership in the research. Parent-respondents to the online SMALLQ ${ }^{\circledR}$ survey.

\section{Compliance with Ethical Standards}

Conflict of interest The research was unfunded and the authors declared no conflict of interest.

Open Access This article is distributed under the terms of the Creative Commons Attribution 4.0 International License (http://creativeco mmons.org/licenses/by/4.0/), which permits unrestricted use, distribution, and reproduction in any medium, provided you give appropriate credit to the original author(s) and the source, provide a link to the Creative Commons license, and indicate if changes were made.

\section{References}

Artino, A. R., Jr., La Rochelle, J. S., Dezee, K. J., \& Gehlbach, H. (2014). Developing questionnaires for educational research: AMEE Guide No. 87. Medical Teacher, 36(6), 463-474. https ://doi.org/10.3109/0142159X.2014.889814.

Buck, D. (2012). The PedsQL ${ }^{\mathrm{TM}}$ as a measure of parent-rated quality of life in healthy UK toddlers: Psychometric properties and cross-cultural comparisons. Journal of Child Health Care, 16(4), 331-338. https://doi.org/10.1177/1367493512448127.

Carson, V., Lee, E. Y., Hewitt, L., Jennings, C., Hunter, S., Kuzik, N., ... Tremblay, M. (2017b). Systematic review of the relationships between physical activity and health indicators in the early years (0-4 years). BMC Public Health, 17(5), 854. https://doi. org/10.1186/s12889-017-4860-0.

Chan, C. M. S., \& Wang, W. C. (2013). Quality of life in overweight and obese young Chinese children: A mixed-method study. Health and Quality of Life Outcomes, 11(1), 33. https://doi. org/10.1186/1477-7525-11-33.

Chaput, J. P., Colley, R. C., Aubert, S., Carson, V., Janssen, I., Roberts, K. C., et al. (2017a). Proportion of preschool-aged children meeting the Canadian 24-Hour movement guidelines and associations with adiposity: Results from the Canadian Health Measures Survey. BMC Public Health, 17(5), 829. https://doi. org/10.1186/s12889-017-4854-y.

Chaput, J. P., Gray, C. E., Poitras, V. J., Carson, V., Gruber, R., Birken, C., ... Tremblay, M. (2017). Systematic review of the relationships between sleep duration and health indicators in the early years (0-4 years). BMC Public Health, 17(5), 855. doi: https://doi.org/10.1186/s12889-017-4850-2.

Chia, M. (2008). Physical inactivity among children and adolescents in Singapore: A paradoxical issue. Acta Kinesiologica, 2, 7-15.

Chia, M. Y. H., Tay, L. Y., \& Chua, T. B. K. (2019). The development of an online surveillance of digital media use in early childhood questionnaire-SMALLQ ${ }^{\mathrm{TM}}$-for Singapore. Montenegrin Journal of Sports Science and Medicine. https://doi. org/10.26773/mjssm.190910.

Children's ENT Centre, KK Women's and Children's Hospital. Sleep (Child). Retrieved July 4, 2019, from https://www.singhealth.com. sg/patient-care/conditions-treatments/sleep-growing-up/overview.

Chung, J., \& Monroe, G. S. (2003). Exploring social desirability bias. Journal of Business Ethics, 44(4), 291-302.

Cliff, D. P., McNeill, J., Vella, S. A., Howard, S. J., Santos, R., Batterham, M., ... de Rosnay, M. (2017). Adherence to 24-Hour Movement Guidelines for the Early Years and associations with social-cognitive development among Australian preschool children. BMC Public Health, 17(5), 857. https://doi.org/10.1186/ s12889-017-4858-7.

De Craemer, M., Verloigne, M., Ghekiere, A., Loyen, A., DargentMolina, P., Brug, J., ... Cardon, G. (2018). Changes in children's television and computer time according to parental education, parental income and ethnicity: A 6-year longitudinal EYHS study. PloS ONE, 13(9), e0203592. https://doi.org/10.1371/journ al.pone. 0203592 .

Department of Statistics, Ministry of Trade and Industry, Singapore. (2010). Census of population 2010 statistical release 1-Demographic characteristics, education, language and religion. Retrieved July 7, 2019, from https://www.singstat.gov.sg/-/media /files/publications/cop2010/census_2010_release1/cop2010sr1 .pdf.

Early Childhood Development Agency. (2019). List of anchor operators. Retrieved June 20, 2019, from https://www.ecda.gov.sg/ Parents/Pages/AOP.aspx.

Harrison, F., Goodman, A., van Sluijs, E. M., Andersen, L. B., Cardon, G., Davey, R., ... \& Pate, R. (2017). Weather and children's physical activity: How and why do relationships vary between countries? International Journal of Behavioral Nutrition and Physical Activity, 14(1), 74. https://doi.org/10.1186/s12966-017-0526-7.

Health Promotion Board. (2013). National physical activity guidelines for youth and adolescents aged up to 18 years: professional guide. Retrieved July 3, 2019, from https://www.academia.edu/10443 994/National_Physical_Activity_Guidelines_for_Children_and Youth.

Hoddinott, S. N., \& Bass, M. J. (1986). The dillman total design survey method. Canadian Family Physician, 32, 2366-2368.

Jenni, O. G., \& O'Connor, B. B. (2005). Children's sleep: An interplay between culture and biology. Pediatrics, 115(Supplement 1), 204-216. https://doi.org/10.1542/peds.2004-0815B.

Lamoureux, E. L., Marella, M., Chang, B., Dirani, M., Kah-Guan, A. E., Chia, A., ... \& Saw, S. M. (2010). Is the pediatric quality of life inventory valid for use in preschool children with refractive errors? Optometry and Vision Science: Official Publication of the American Academy of Optometry, 87(11), 813. https://doi. org/10.1097/opx.0b013e3181f6fb84.

Legislation Division, Attorney-General's Chambers, Singapore. (2012). Personal Data Protection Act 2012. Retrieved September 29, 2019, from https://sso.agc.gov.sg/Act/PDPA2012.

Liu, X., Liu, L., \& Wang, R. (2003). Bed sharing, sleep habits, and sleep problems among Chinese school-aged children. Sleep, 26(7), 839-844. https://doi.org/10.1093/sleep/26.7.839.

Mielke, G. I., Brown, W. J., Nunes, B. P., Silva, I. C., \& Hallal, P. C. (2017). Socioeconomic correlates of sedentary behavior in adolescents: Systematic review and meta-analysis. Sports Medicine, 47(1), 61-75. https://doi.org/10.1007/s40279-016-0555-4. 
New, R. S., \& Richman, A. L. (1996). Maternal beliefs and infant care practices in Italy and the United States. In S. Harkness \& C. M. Super (Eds.), Parents' cultural belief systems: Their origins, expressions, and consequences (pp. 385-404). New York: Guilford Press.

O’Connor, T. M., Hingle, M., Chuang, R. J., Gorely, T., Hinkley, T., Jago, R., ... Thompson, D. A. (2013). Conceptual understanding of screen media parenting: Report of a working group. Childhood Obesity, 9(s1), S-110. https://doi.org/10.1089/chi.2013.0025.

Palmer, L., Johnston, S. S., Rousculp, M. D., Chu, B. C., Nichol, K. L., \& Mahadevia, P. J. (2012). Agreement between internetbased self-and proxy-reported health care resource utilization and administrative health care claims. Value in Health, 15(3), 458-465. https://doi.org/10.1016/j.jval.2011.12.015.

PedsQL ${ }^{\text {TM }}$, Copyright (C) 1998 JW Varni, Ph.D. All rights reserved.

Poitras, V. J., Gray, C. E., Janssen, X., Aubert, S., Carson, V., Faulkner, G., ... Tremblay, M. (2017). Systematic review of the relationships between sedentary behaviour and health indicators in the early years (0-4 years). BMC Public Health, 17(5), 868. https:// doi.org/10.1186/s12889-017-4849-8.

Prensky, M. (2001). Digital natives, digital immigrants part 1. On the Horizon, 9(5), 1-6. https://doi.org/10.1108/10748120110424816.

Puder, J., Pinto, A. M., Bonvin, A., Bodenman, P., Munsch, S., Kriemler, S., et al. (2013). Health-related quality of life in migrant preschool children. BMC Public Health, 13(1), 384. https://doi. org/10.1186/1471-2458-13-384.

Short, M. E., Goetzel, R. Z., Pei, X., Tabrizi, M. J., Ozminkowski, R. J., Gibson, T. B., ... \& Wilson, M. G. (2009). How accurate are self-reports? An analysis of self-reported healthcare utilization and absence when compared to administrative data. Journal of Occupational and Environmental Medicine/American College of Occupational and Environmental Medicine, 51(7), 786. https:// doi.org/10.1097/JOM.0b013e3181a86671.
Steger, B. (2003). Negotiating sleep patterns in Japan. In B. Steger \& L. Brunt (Eds.), Night-time and sleep in Asia and the West: Exploring the dark side of life (pp. 65-86). London: Routledge Curzon.

Stiglic, N., \& Viner, R. M. (2019). Effects of screentime on the health and well-being of children and adolescents: A systematic review of reviews. British Medical Journal Open, 9(1), e023191. https:// doi.org/10.1136/bmjopen-2018-023191.

Tamana, S. K., Ezeugwu, V., Chikuma, J., Lefebvre, D. L., Azad, M. B., Moraes, T. J., .. \& \& Dick, B. D. (2019). Screen-time is associated with inattention problems in preschoolers: Results from the CHILD birth cohort study. PloS ONE, 14(4), e0213995. https:// doi.org/10.1371/journal.pone.0213995.

Varni, J. W., Burwinkle, T. M., Seid, M., \& Skarr, D. (2003). The Ped$\mathrm{sQL}^{\mathrm{TM}} * 4.0$ as a pediatric population health measure: Feasibility, reliability, and validity. Ambulatory Pediatrics, 3(6), 329-341. https://doi.org/10.1367/1539-4409(2003)003\%3c0329:TPAAP $\mathrm{P} \% 3 \mathrm{e} 2.0 . \mathrm{CO} ; 2$.

Varni, J. W., Seid, M., \& Rode, C. A. (1999). The PedsQL ${ }^{\mathrm{TM}}$ : Measurement model for the pediatric quality of life inventory. Medical Care, 37, 126-139.

World Health Organisation. (2019). Guidelines on physical activity, sedentary behaviour and sleep for children under 5 years of age. Retrieved July 3, 2019, from https://apps.who.int/iris/handl e/10665/311664. Licence: CC BY-NC-SA 3.0 IGO.

Publisher's Note Springer Nature remains neutral with regard to jurisdictional claims in published maps and institutional affiliations. 